Comment. Math. Helv. 74 (1999) 306-321

(C) 1999 Birkhäuser Verlag, Basel

0010-2571/99/020306- $16 \$ 1.50+0.20 / 0$

Commentarii Mathematici Helvetici

\title{
Projectively equivalent metrics, exact transverse line fields and the geodesic flow on the ellipsoid
}

Serge Tabachnikov

\begin{abstract}
We give a new proof of the complete integrability of the geodesic flow on the ellipsoid (in Euclidean, spherical or hyperbolic space). The proof is based on the construction of a metric on the ellipsoid whose non-parameterized geodesics coincide with those of the standard metric. This new metric is induced by the hyperbolic metric inside the ellipsoid (Klein's model).
\end{abstract}

Mathematics Subject Classification (1991). 53.

Keywords. Riemannian and Finsler metrics, symplectic and contact structures, geodesic flow, exact transverse line fields.

\section{Introduction}

The geodesic flow on the ellipsoid is one of the most popular examples of a completely integrable dynamical system (for the triaxial ellipsoid integrability was established by Jacobi in 1838). A number of proofs of integrability is known: by separation of variables ( Jacobi's original approach), by confocal quadrics, by isospectral deformations. An interested reader is referred to $[2,3,4,7,9,10,14]$.

In this paper we give still another proof of complete integrability of the geodesic flow on the ellipsoid. Our proof is based on the simple remark: if a dynamical system (with continuous or discrete time) on a $2 n$-dimensional manifold possesses two invariant differential 2-forms one of which is nondegenerate then the system has $n$ invariant functions. Namely, let the invariant forms be $\omega_{1}$ and $\omega_{2}$ where $\omega_{1}$ is nondegenerate. Then the functions

$$
\frac{\omega_{2}^{i} \wedge \omega_{1}^{n-i}}{\omega_{1}^{n}}, \quad i=1, \ldots, n
$$

are integrals (not necessarily independent). Alternatively, consider the $(1,1)$ tensor field $E$ relating the two forms: $\omega_{2}(u, v)=\omega_{1}(E u, v)$ for every tangent vectors $u$ and $v$. Then the eigenvalues of $E$ are invariant functions, and these integrals are functionally dependent on the previous ones. 
A much deeper result (which we do not use) is that if $\omega_{1}$ and $\omega_{2}$ are Poisson compatible symplectic forms then these integrals Poisson commute with respect to both forms - see, e.g., [5, 12] concerning bihamiltonian formalism.

In Section 2 we slightly modify and apply the above general observation as follows. Consider the geodesic flow of a Finsler metric on a manifold $M$. One identifies the tangent and cotangent bundles via the Legendre transformation and considers the differential 1-form $\lambda$ corresponding to the Liouville form on $T^{*} M$ and the respective symplectic structure $\omega=d \lambda$. The geodesic flow is a Hamiltonian flow on $T M$ whose Hamiltonian function is identified with the Lagrangian defining the Finsler metric. Let $S \subset T M$ be the hypersurface consisting of Finsler unit vectors. The leaves of the characteristic foliation $\eta$ on $S$ are identified with nonparameterized trajectories of the geodesic flow.

Two Finsler metrics on $M$ are called projectively equivalent if, up to reparameterization, their geodesics coincide. We show that if there exist two projectively equivalent Finsler metrics on $M^{n}$ then the geodesic flow of either metric has $n$ invariant functions. If the metrics are generic enough then these integrals are functionally independent.

The argument goes as follows. Given two projectively equivalent Finsler metrics on $M$, consider the transformation $\phi$ of $T M$ that rescales the tangent vectors and takes the first unit vector hypersurface $S_{1}$ to the second one, $S_{2}$. Then $\phi$ takes the characteristic foliation $\eta_{1}$ to $\eta_{2}$. Therefore the symplectic forms $\omega_{1}$ and $\phi^{*}\left(\omega_{2}\right)$ have the same characteristic foliation $\eta_{1}$ on $S_{1}$ and both forms are holonomy invariant along $\eta_{1}$. It follows that the functions

$$
\frac{\lambda_{1} \wedge \omega_{1}^{n-1-i} \wedge \omega_{2}^{i}}{\lambda_{1} \wedge \omega_{1}^{n-1}}, \quad i=1, \ldots, n-1
$$

are integrals of the geodesic flow of the first Finsler metric, the $n$-th integral being the Lagrangian (once again one may also consider the spectrum of the field of the linear transformations relating the two forms).

It is an interesting problem to describe the pairs of projectively equivalent Finsler metrics for which the constructed integrals Poisson commute with respect to the symplectic structure $\omega_{1}$. In particular, when are the forms $\omega_{1}$ and $\phi^{*}\left(\omega_{2}\right)$ Poisson compatible?

We apply this construction to the geodesic flow on the ellipsoid. One of the metrics is, of course, the Euclidean metric in the ambient space restricted to the ellipsoid. The other metric is provided by the following general construction discussed in Section 3.

Consider a smooth hypersurface $M \subset \mathbf{R}^{n}$ equipped with a smooth transverse line field $\xi$. A smooth curve $\gamma$ on $M$ is called a $\xi$-geodesic if at every point $x \in \gamma$ the 2-plane generated by the tangent line to $\gamma$ and the line $\xi(x)$ is second-order tangent to $\gamma$; in other words, the osculating 2-plane of a $\xi$-geodesic at every point $x$ contains the line $\xi(x)$. In particular, if $\xi$ consists of the Euclidean normals then a $\xi$-geodesic is the usual geodesic line on $M$. We show that if $M$ is an 
ellipsoid and $\xi$ is a so-called exact field (introduced and studied in $[15,16,17]$ and defined in Section 3) then there exists a metric on $M$ whose geodesics coincide with $\xi$-geodesics (this result was conjectured in [15]). Since the field of Euclidean normals to a hypersurface is exact one obtains the second metric on the ellipsoid, projectively equivalent to the Euclidean one.

This second metric comes from the hyperbolic metric inside the ellipsoid considered as the Klein model of the hyperbolic space $\mathbf{H}^{n}$; the ellipsoid plays the role of the sphere at infinity. Namely, there exists a one-parameter family of closed hypersurfaces in $\mathbf{H}^{n}$ that are orthogonal, in the hyperbolic sense, to the lines from the field $\xi$. These surfaces have the metrics induced from $\mathbf{H}^{n}$, and the desired metric on the ellipsoid is the limit of these, appropriately renormalized, metrics as the hypersurfaces tend, in the Euclidean sense, to the sphere at infinity.

This construction is closely related with a result from [15] concerning the billiard inside the ellipsoid. The billiard transformation is a map of the space of oriented lines (rays) intersecting the billiard table; this map preserves the symplectic structure on the space of rays associated with the Euclidean metric (see, e.g., [18]). It was observed in [15] that if the billiard table is an ellipsoid then the billiard map also preserves the symplectic structure on the space of rays associated with the hyperbolic metric inside the ellipsoid. This observation gives a proof of complete integrability of the billiard map.

In Section 4 we compute the integrals for the geodesic flow on the ellipsoid provided by the preceding constructions. Remarkably, we obtain precisely the classical integrals (as given in $[8,9,10]$ ).

In Section 5 we show that the same techniques apply to the geodesic flow on the ellipsoid in the spherical or hyperbolic space. These flows are completely integrable - see [20]; our method provides a new proof.

We also describe the Riemannian metrics in a domain $D \subset \mathbf{R}^{n}$ with the property that for every smooth hypersurface $M \subset D$ there exists a smooth transverse line field $\xi$ along $M$ such that the geodesics on $M$ are the $\xi$-geodesics. It turns out that such a metric is a metric of constant curvature whose geodesics are straight lines, that is, the Euclidean, spherical or hyperbolic one; the respective transverse line field consists of the normals to a hypersurface.

It would be interesting to describe the Finsler metrics with the same property; at the present writing we do not know whether there are any which are not Riemannian. Such a Finsler metric would be a candidate for application of the above described techniques, and one might expect its geodesic flow on the ellipsoid to be integrable.

In the Appendix we prove Hamel's characterization of Finsler metrics in a domain in linear space that are projectively equivalent to the Euclidean ones.

We use vector notation throughout the paper: if $x, u \in \mathbf{R}^{n}$ and $L$ is a function on $\mathbf{R}^{n}$ then

$$
\begin{gathered}
x u=x_{1} u_{1}+\ldots+x_{n} u_{n}, \quad u d x=u_{1} d x_{1}+\ldots+u_{n} d x_{n} \\
d x \wedge d u=d x_{1} \wedge d u_{1}+\ldots+d x_{n} \wedge d u_{n}, \quad \partial L / \partial u=L_{u}=\left(L_{u_{1}}, \ldots, L_{u_{n}}\right),
\end{gathered}
$$




$$
u(L)=u L_{u}=u_{1} L_{u_{1}}+\ldots+u_{n} L_{u_{n}} \text { etc. }
$$

\section{Projectively equivalent Finsler metrics}

Start with a description of relevant facts about Finsler metrics (see, e.g., [3, 13]).

Finsler geometry describes the propagation of light in an inhomogeneous anisotropic medium. This means that the velocity of light depends on the point and the direction. There are two equivalent descriptions of this process corresponding to the Lagrangian and the Hamiltonian approaches in classical mechanics; we will focus on the former.

A Finsler metric on a manifold $M$ is described by a smooth field of strictly convex smooth hypersurfaces, containing the origin (and not necessarily centrallysymmetric), in the tangent space at each point. These hypersurfaces are called indicatrices. The indicatrix consists of the Finsler unit vectors and plays the role of the unit sphere in Riemannian geometry.

Equivalently, a Finsler metric is determined by a smooth nonnegative fiberwise convex Lagrangian function $L$ on the tangent bundle $T M$ whose unit level hypersurface $S$ intersects each fiber of $T M$ along the indicatrix. Unless otherwise specified, we assume that $L$ is fiber-wise homogeneous of degree 2 .

A Finsler geodesic is an extremal of the Lagrangian $L$. The Finsler geodesic flow $v$ is a flow in $T M$ in which the foot point of a tangent vector moves along a Finsler geodesic, the vector remains tangent to this geodesic and has a constant Finsler length.

The Lagrangian $L$ determines a diffeomorphism, called the Legendre transformation, between the tangent and cotangent bundles. If $(x, u), x \in M, u \in T_{x} M$ is a tangent vector and $(x, p), x \in M, p \in T_{x}^{*} M$ is a cotangent one then the Legendre transformation is given by the formula: $p=L_{u}$.

Denote by $\lambda$ the differential 1-form in $T M$ corresponding to the Liouville form in $T^{*} M$; one has: $\lambda=L_{u} d x$. Let $\omega=d \lambda$; then $\omega$ is a symplectic form in $T M$. The flow $v$ is a Hamiltonian flow with respect to this symplectic structure, the Hamilton function being $L$. One has: $\lambda(v)=2 L$ and $i_{v} \omega=-d L=\mathcal{L}_{v} \lambda$ (where $\mathcal{L}_{v}$ is the Lie derivative).

The unit level hypersurface $S \subset T M$ is foliated by the trajectories of the geodesic flow. These trajectories coincide with the leaves of the characteristic foliation $\eta$ generated by the kernels of $\omega$ restricted to $S$. Thus the leaves of $\eta$ are identified with non-parameterized Finsler geodesics. The 1-form $\lambda$ determines a contact structure on $S$.

Let two projectively equivalent Finsler metrics be given on $M^{n}$; we use the subscript $i=1,2$ to indicate that an object relates to the first or the second metric. Consider the diffeomorphism $\phi$ of $T M$ given by the formula:

$$
\phi:(x, u) \rightarrow(X, U) \quad \text { where } \quad X=x, U=\left(L_{1}(u) / L_{2}(u)\right)^{1 / 2} u .
$$


This transformation has the following properties.

Lemma 2.1. (i) $\phi\left(S_{1}\right)=S_{2}$.

(ii) The 2-forms $\omega_{1}$ and $\phi^{*} \omega_{2}$ have the same characteristic foliation $\eta_{1}$ on $S_{1}$ and both are holonomy invariant along the leaves of this foliation.

Proof. Since $L_{1}(u)=1$ on $S_{1}$, one has: $L_{2}(U)=\left(L_{1}(u) / L_{2}(u)\right) L_{2}(u)=1$, and the first claim follows. Since the Finsler metrics are projectively equivalent, $\phi$ takes the leaves of $\eta_{1}$ to those of $\eta_{2}$. Since $\eta_{2}$ is the characteristic foliation of $\omega_{2}$ on $S_{2}$ the characteristic foliation of $\phi^{*} \omega_{2}$ on $S_{1}$ is $\eta_{1}$. If $v$ is a vector field tangent to $\eta_{1}$ then $i_{v} \omega_{1}=0$ and $L_{v}\left(\omega_{1}\right)=i_{v} d \omega_{1}+d i_{v} \omega_{1}=0$ on $S_{1}$. The same applies to $\phi^{*} \omega_{2}$, and the second claim is proved.

It follows that the functions invariantly associated with the forms $\omega_{1}$ and $\phi^{*} \omega_{2}$ are constant on the leaves of $\eta_{1}$, that is, are integrals of the geodesic flow of the first Finsler metric.

One way to obtain such functions is as follows. Let $E$ be the $(1,1)$-tensor field relating the two forms: $\phi^{*} \omega_{2}(u, v)=\omega_{1}(E u, v)$ for every tangent vectors $u$ and $v$. Since $\eta_{1}$ is the common characteristic foliation of the two forms, $E$ preserves the tangent directions to $\eta_{1}$. It follows that the $n-1$ eigenvalues of the linear transformations induced by $E$ on the fibers of the normal bundle $T S / \eta_{1}$ are integrals of the geodesic flow; still another integral is, of course, the Lagrangian $L_{1}$.

Another construction of invariant functions goes as follows. The integrals constructed below are functionally dependent on the above ones.

Lemma 2.2. The functions

$$
f_{i}=\frac{\lambda_{1} \wedge \omega_{1}^{n-1-i} \wedge \phi^{*} \omega_{2}^{i}}{\lambda_{1} \wedge \omega_{1}^{n-1}}, \quad i=1, \ldots, n-1
$$

are constant along the leaves of $\eta_{1}$.

Proof. Since $\lambda_{1}$ is a contact form the denominator does not vanish. Similarly to Lemma 2.1, $L_{v_{1}}\left(\lambda_{1}\right)=0$ and $L_{v_{1}}\left(\omega_{1}\right)=L_{v_{1}}\left(\phi^{*} \omega_{2}\right)=0$ on $S_{1}$. It follows that $v_{1}\left(f_{i}\right)=0$ for every $i$.

Notice that the symplectic form $\phi^{*} \omega_{2}$ is not necessarily $v_{1}$-invariant on the whole $T M$. We modify the form $\omega_{2}$ as follows.

Lemma 2.3. The kernel of the 2-form $\omega_{2}^{\prime}=d\left(L_{2}^{-1 / 2} \lambda_{2}\right)$ contains the vector $v_{2}$, and for every function $g$ this 2 -form is invariant on TM under the flow $g v_{2}$. 
Proof. One has:

$$
\omega_{2}^{\prime}=(1 / 2) L_{2}^{-3 / 2}\left(2 L_{2} d \lambda_{2}-d L_{2} \wedge \lambda_{2}\right)
$$

Therefore

$$
\begin{aligned}
i_{v_{2}} \omega_{2}^{\prime} & =(1 / 2) L_{2}^{-3 / 2}\left(2 L_{2} i_{v_{2}} d \lambda_{2}+\lambda_{2}\left(v_{2}\right) d L_{2}\right) \\
& =(1 / 2) L_{2}^{-3 / 2}\left(-2 L_{2} d L_{2}+2 L_{2} d L_{2}\right)=0
\end{aligned}
$$

It follows that $g v_{2} \in \operatorname{ker} \omega_{2}^{\prime}$. Since $\omega_{2}^{\prime}$ is closed, the Cartan's formula for the Lie derivative implies that $\omega_{2}^{\prime}$ is $\left(g v_{2}\right)$-invariant. The lemma is proved.

Since the map $\phi$ takes $L_{1}$ to $L_{2}$ and the vector field $\phi_{*}\left(v_{1}\right)$ is equal, up to a functional multiplier, to $v_{2}$ we obtain the following corollary.

Corollary 2.4. The 2-form $\phi^{*} \omega_{2}^{\prime}=d\left(L_{1}^{-1 / 2} \phi^{*} \lambda_{2}\right)$ is invariant, along with $\omega_{1}$, under the geodesic flow of the first Finsler metric on TM.

\section{Exact transverse line fields along the sphere}

Let $M \subset \mathbf{R}^{n}$ be a smooth hypersurface equipped with a smooth transverse line field $\xi$. Denote by $n(x), x \in M$ the unit normal vector field along $M$. Let $v$ be the vector field along $\xi$ normalized so that $v(x) n(x)=1$ for all $x \in M$.

Definition. A transverse field $\xi$ is called exact if the 1 -form $v d n$ on $M$ is exact.

Clearly, the field of Euclidean normals is exact. Exact fields enjoy many properties of the Euclidean normals. For example, the following generalization of the classical 4-vertex theorem holds (see [19]): given a generic exact transverse line field $\xi$ along a closed convex smooth plane curve $\gamma$, the envelope of the 1-parameter family of lines $\xi(x), x \in \gamma$ has at least 4 cusp singularities.

Below we list a few relevant properties of exact transverse line fields - see $[15$, 16]:

(i) Although defined in Euclidean terms, exactness is a projective property. Namely, let $\xi$ be an exact transverse line field along $M \subset \mathbf{R}^{n}$, and $F$ be a projective transformation of $\mathbf{R}^{n}$ whose domain contains $M$. Then the line field $D F(\xi)$ along $F(M)$ is also exact.

(ii) Let $f$ be a smooth function on the unit sphere $\mathbf{S}^{n-1}$. Then the line field generated by the vector field $v(x)=x+\operatorname{grad} f(x), x \in \mathbf{S}^{n-1}$ is exact. Every exact field along the unit sphere is obtained this way.

(iii) Let $\xi$ be an exact transverse line field along a sphere. Identifying the interior of the sphere with the hyperbolic space $\mathbf{H}^{n}$ (Klein's model), one has an imbedding of the sphere to the space of oriented lines in $\mathbf{H}^{n}$ : to a point $x \in S^{n-1}$ 
there corresponds the line $\xi(x)$, oriented outward. Let $L$ be the image of this map. Then $L$ is a Lagrangian submanifold of the space of oriented lines in $\mathbf{H}^{n}$ with its canonical symplectic structure, associated with the hyperbolic metric, and if $n \geq 3$ this condition is equivalent to exactness. It follows that if $\xi$ is exact then the lines from this field are the hyperbolic normals to a one-parameter family of equidistant closed hypersurfaces in $\mathbf{H}^{n}$. In view of Property (i), an analogous property holds for the ellipsoid.

(iv) Let $g$ be a Riemannian metric of constant positive or negative curvature in a domain $D \subset \mathbf{R}^{n}$ whose geodesics are straight lines. Then, for every hypersurface $M \subset D$ the field of $g$-normals to $M$ is an exact line field.

Let $\xi$ be an exact transverse line field along the unit sphere. According to Property (ii), $\xi$ is generated by the vector field $v(x)=x+\operatorname{grad} f(x)$ where $f$ is a smooth function on the sphere. Let $(x, u), x \in \mathbf{S}^{n-1}, u \in T_{x} \mathbf{S}^{n-1}$ be coordinates in $T \mathbf{S}^{n-1}$. We ask whether there exists a metric on the sphere whose geodesics are the $\xi$-geodesics. The affirmative answer is provided by the next theorem.

Theorem 3.1. The geodesics of the metric $L(x, u)=u^{2} e^{-2 f(x)} / 2$ are the $\xi$ geodesics.

Proof. One wants to show that if $x(t)$ is a geodesic of $L$ then for every $t$ the acceleration vector $x^{\prime \prime}(t)$ belongs to the plane spanned by $x^{\prime}(t)$ and $v(x(t))$.

The Euler-Lagrange equation with constraints reads (see, e.g., [3]):

$$
L_{u u} x^{\prime \prime}+L_{u x} x^{\prime}-L_{x}=C x
$$

where $C$ is an unknown function and $x^{\prime}=u$. One easily computes:

$$
L_{x}=-u^{2} e^{-2 f} \operatorname{grad} f, L_{u}=e^{-2 f} u, L_{u x}=-2 e^{-2 f} u \otimes \operatorname{grad} f, L_{u u}=e^{-2 f} I d,
$$

where $u \otimes \operatorname{grad} f$ is the endomorphism

$$
(u \otimes \operatorname{grad} f)_{i j}=u_{i} \frac{\partial f}{\partial x_{j}} .
$$

Thus the Euler-Lagrange equation is:

$$
e^{-2 f}\left(x^{\prime \prime}-2 u(f) u+u^{2} \operatorname{grad} f\right)=C x,
$$

where $u(f)$ is the directional derivative of the function $f$. To find $C$, dot-multiply by $x$ and use the fact that $u x=0$ and $x \cdot \operatorname{grad} f=0$; one finds that

$$
C=e^{-2 f} x^{\prime \prime} x=-e^{-2 f} u^{2},
$$

the last equality obtained by differentiating the identity $x u=0$. Therefore

$$
x^{\prime \prime}-2 u(f) u+u^{2} \operatorname{grad} f=-u^{2} x, \quad \text { hence } \quad x^{\prime \prime}=2 u(f) u+u^{2} v(x) .
$$


It follows that the acceleration $x^{\prime \prime}$ is a linear combination of the velocity $x^{\prime}$ and the transverse vector $v(x)$. The theorem is proved.

It would be interesting to describe all pairs $(M, \xi)$ where $M$ is a hypersurface and $\xi$ is a transverse line field for which there exists a Lagrangian on $M$ whose extremals are the $\xi$-geodesics.

Next we discuss the relation between the Lagrangian from the previous theorem and the above mentioned Property (iii) of exact transverse line fields. We use the notation introduced prior to Theorem 3.1.

The idea is as follows. Consider the one-parameter family of equidistant closed hypersurfaces inside the sphere which are orthogonal with respect to the hyperbolic metric to the lines of the exact transverse field $\xi$ along $\mathbf{S}^{n-1}$. Let $N$ be such a hypersurface; it has the metric induced from the ambient hyperbolic space. We want to consider the limit of these metrics as $N$ tends to $\mathbf{S}^{n-1}$. We need, however, to renormalize the metrics, otherwise the limit will be infinite.

To this end let $N$ be a hypersurface which is, in the Euclidean sense, infinitesimally close to the unit sphere. Denote by $y \in N$ the point that lies on the line $\xi(x)$; the correspondence $\psi: x \rightarrow y$ is a diffeomorphism from $\mathbf{S}^{n-1}$ to $N$. One has:

$$
y=x-\epsilon h(x) v(x)+O\left(\epsilon^{2}\right)
$$

where $h$ is a positive function on the sphere and $\epsilon$ denotes an infinitesimal parameter. Let $g_{\epsilon}$ be the metric on the sphere induced by $\psi$ from the metric on $N$ and rescaled by the factor $\epsilon$.

Theorem 3.2. The metric from Theorem 3.1 is equal, up to a constant, to $\lim _{\epsilon \rightarrow 0} g_{\epsilon}$.

Proof. To start with, the hyperbolic metric inside the unit ball is given, in Euclidean terms, by the formula:

$$
L_{H}(y, w)=\frac{w^{2}}{1-y^{2}}+\frac{(w y)^{2}}{\left(1-y^{2}\right)^{2}}
$$

where $y$ is a point inside the ball, $w$ is a tangent vector and multiplication is the Euclidean scalar product (see, e.g., [15]). Respectively, the hyperbolic scalar product at point $y$ is

$$
<w_{1}, w_{2}>=\frac{w_{1} w_{2}}{1-y^{2}}+\frac{\left(w_{1} y\right)\left(w_{2} y\right)}{\left(1-y^{2}\right)^{2}}
$$

Recall that $\xi$ is generated by the vector field $v(x)=x+\operatorname{grad} f(x)$ and that $x v(x)=1$. Write the unknown function $h(x)$ as $\exp \phi(x)$. Let $u \in T_{x} \mathbf{S}^{n-1}$ be a tangent vector and $w=D \psi(u)$. The function $\phi$ is determined by the condition that the lowest term in $\epsilon$ in the scalar product $\langle w, v\rangle$ vanishes for every $u$. 
It is straightforward to compute that

$w=u-\epsilon e^{\phi}(u(\phi) v+u(v))+O\left(\epsilon^{2}\right), 1-y^{2}=2 \epsilon e^{\phi}+O\left(\epsilon^{2}\right), y v=1-\epsilon e^{\phi} v^{2}+O\left(\epsilon^{2}\right)$.

It follows that

$$
y w=-\epsilon e^{\phi}(u(\phi)+x u(v)+u v)+O\left(\epsilon^{2}\right) \text { and } v w=u v+O(\epsilon) .
$$

Take the directional derivative of the identity $x v=1$ and use the tautological identity $u(x)=u$ to obtain: $u v+x u(v)=0$. In addition, $u v=u \cdot \operatorname{grad} f=u(f)$; therefore

$$
y w=-\epsilon e^{\phi} u(\phi)+O\left(\epsilon^{2}\right) \text { and } v w=u(f)+O(\epsilon) .
$$

Hence the lowest term in $\langle w, v\rangle$ is

$$
\frac{2 u(f)-u(\phi)}{4 \epsilon e^{\phi}} .
$$

Equating to zero for every $u$ one finds: $\phi=2 f+$ const.

Finally, $g_{\epsilon}(x, u)=\epsilon L_{H}(y, w)$ where $(y, w)$ is related to $(x, u)$ as above. The formula for $L_{H}$ and the preceding computations imply that

$$
g_{\epsilon}(x, u)=\text { Const } \frac{1}{2} u^{2} e^{-2 f(x)}+O(\epsilon)
$$

and the result follows.

Remark. We give an explicit parameterization of the equidistant hypersurfaces orthogonal to the lines of the field $\xi$ : these hypersurfaces, indexed by a parameter $\epsilon$ which is an arbitrary positive real number, are the loci of the points

$$
y=x-\frac{2 \epsilon v(x) e^{2 f(x)}}{2+\epsilon v^{2}(x) e^{2 f(x)}}, \quad x \in \mathbf{S}^{n-1} .
$$

This formula is a result of a direct computation similar to the one in the proof of the above theorem; we do not reproduce this computation here. The previous result concerned the case of an infinitesimal $\epsilon$.

\section{Integrals of the geodesic flow on the ellipsoid}

In this section we compute the integrals of the geodesic flow on the ellipsoid provided by the construction from Section 2 .

Let $E \subset \mathbf{R}^{n}$ be the ellipsoid given by the equation $\sum a_{i} y_{i}^{2}=1$. Let $(y, v), y \in$ $E, v \in T_{y} E$ be coordinates in the tangent bundle $T E$. Consider the linear transformation $A$ that takes the ellipsoid to the unit sphere $\mathbf{S}^{n-1}$ and extend $A$ to $T E$ :

$$
A:(y, v) \rightarrow(x, u) ; \quad x_{i}=\sqrt{a_{i}} y_{i}, u_{i}=\sqrt{a_{i}} v_{i}
$$


The geodesics on $E$ are transformed by $A$ to the geodesics of the following metric on the sphere:

$$
L_{1}(x, u)=\frac{1}{2} v^{2}=\frac{1}{2} \sum \frac{u_{i}^{2}}{a_{i}} .
$$

Let $n(y)$ be a normal vector to $E$ at point $y$. For every geodesic through $y$ its osculating 2-plane contains $n(y)$. Therefore the $A$-images of the geodesics on the sphere are $\xi$-geodesics where the transverse line field $\xi$ is generated by the vectors $A(n)$. Due to projective invariance of exactness (Property (i) in Section 3) the field $\xi$ is exact. We compute the metric associated with this field according to Theorem 3.1.

Lemma 4.1. This metric is given by the formula:

$$
L_{2}(x, u)=\frac{1}{2} \frac{u^{2}}{\sum a_{i} x_{i}^{2}}
$$

Proof. We may take $n(y)$ to have the components $a_{i} y_{i}$; then $A(n)(x)$ has the components $a_{i} x_{i}$. Rescale this vector to the vector $v(x)$ with the components $a_{i} x_{i} / \sum a_{j} x_{j}^{2}$; then $x v(x)=1$. One easily verifies that $v(x)=x+\operatorname{grad} f(x)$ where $f$ is the following function on the sphere: $f(x)=\ln \left(\sum a_{i} x_{i}^{2}\right) / 2$. It follows that the metric provided by Theorem 3.1 is

$$
\frac{1}{2} u^{2} e^{-2 f(x)}=\frac{1}{2} \frac{u^{2}}{\sum a_{i} x_{i}^{2}}
$$

The lemma is proved.

Thus $L_{1}$ and $L_{2}$ are projectively equivalent metrics. We are ready to apply the construction from Section 2.

Introduce the following notation:

$$
\alpha=\sum \frac{u_{i}^{2}}{a_{i}}, \beta=\sum a_{i} x_{i}^{2} ; \quad \text { then } L_{1}=\frac{1}{2} \alpha, L_{2}=\frac{1}{2} \frac{u^{2}}{\beta} .
$$

Using the notation from Section 2, first compute the 1-forms on $T \mathbf{S}^{n-1}$ corresponding to both Lagrangians:

$$
\lambda_{1}=\sum \frac{u_{i} d x_{i}}{a_{i}}, \quad \lambda_{2}=\frac{u d x}{\beta} .
$$

Next, the map $\phi$ that takes the unit level hypersurface of $L_{1}$ to that of $L_{2}$ is the rescaling by the factor of $\sqrt{\alpha \beta / u^{2}}$. It follows that

$$
\phi^{*}\left(\lambda_{2}\right)=\sqrt{\frac{\alpha}{u^{2} \beta}} u d x ; \text { let } \gamma=\left(u^{2} \beta\right)^{-1 / 2} .
$$


Therefore

$$
\omega_{1}=\sum \frac{d u_{i} \wedge d x_{i}}{a_{i}}, \quad \phi^{*} \omega_{2}^{\prime}=d\left(L_{1}^{-1 / 2} \phi^{*} \lambda_{2}\right)=\sqrt{2} \gamma(d u \wedge d x+d \ln \gamma \wedge(u d x)) .
$$

We drop the subscript and denote $\omega_{1}$ by $\omega$; let also $\Omega=d u \wedge d x+d \ln \gamma \wedge(u d x)$.

We need an explicit formula for the geodesic flow $V$ of $L_{1}$.

Lemma 4.2. One has:

$$
V=\sum\left(u_{i} \frac{\partial}{\partial x_{i}}-\frac{u^{2}}{\beta} a_{i} x_{i} \frac{\partial}{\partial u_{i}}\right) .
$$

Proof. The tangent vectors to $T \mathbf{S}^{n-1}$ belong in the kernels of the 1-forms $x d x$ and $u d x+x d u$; one sees that the above $V$ annihilates both forms. Next, the geodesic flow is the Hamiltonian flow of $L_{1}$ with respect to the symplectic structure $\omega$; and indeed, the above $V$ satisfies the formula: $i_{V} \omega=-d L_{1}$. The lemma is proved.

We also make an observation that is verified by a direct computation.

Lemma 4.3. $V(\gamma)=0$.

Thus, in addition to $\alpha$, the quadratic function $u^{2} \beta$ is an integral of the geodesic flow $V$; being expressed in terms of the original ellipsoid, this is the classical Joachimsthal integral. It follows that $\Omega$ is an invariant form (neither closed nor non-degenerate). This form is easily computed:

$$
\Omega=d u \wedge d x-\frac{(u d u) \wedge(u d x)}{u^{2}}-\frac{(x d u) \wedge\left(\sum a_{i} x_{i} d x_{i}\right)}{\beta} .
$$

We are ready to compute integrals of the geodesic flow from the invariant forms $\omega$ and $\Omega$.

Consider the $V$-invariant 2-form $\omega_{t}=t^{-1} \omega+\Omega$. Then the function $f_{t}=$ $\omega_{t}^{n-1} / \omega^{n-1}$ is an integral of the flow $V$ for every $t$.

Consider $T \mathbf{S}^{n-1}$ as a submanifold in $T \mathbf{R}^{n}$; then the 1 -forms $x d u+u d x$ and $x d x$ vanish on $T \mathbf{S}^{n-1}$. The computation of the functions $f_{t}$ simplifies if one wedgemultiplies the numerator and denominator by the 2 -form $(x d u+u d x) \wedge(x d x)$ and considers the resulting $2 n$-forms in $T \mathbf{R}^{n}$. Moreover, wedge-multiplying the numerator by $u d x \wedge x d x$ yields zero because it contributes $n+1$ factors of the type $d x_{i}$. Also note that wedge-multiplying the numerator by $x d u$ annihilates the term $(x d u) \wedge\left(\sum a_{i} x_{i} d x_{i}\right)$ in $\Omega$. Furthermore, since $((u d u) \wedge(u d x))^{2}=0$, the function $f_{t}$ is equal to the ratio of the two volume forms $W_{1}$ and $W_{2}$ in $T \mathbf{R}^{n}$ where $W_{1}=$

$$
\left(\left(\sum \frac{d u_{i} \wedge d x_{i}}{b_{i}}\right)^{n-1}-\frac{(n-1)}{u^{2}}\left(\sum \frac{d u_{i} \wedge d x_{i}}{b_{i}}\right)^{n-2} \wedge(u d u) \wedge(u d x)\right) \wedge(x d u) \wedge(x d x),
$$




$$
W_{2}=\left(\sum \frac{d u_{i} \wedge d x_{i}}{a_{i}}\right)^{n-1} \wedge(x d u) \wedge(x d x)
$$

and

$$
b_{i}=\frac{a_{i} t}{1+a_{i} t}=1-\frac{1}{1+a_{i} t} .
$$

Let $W_{0}$ be the standard volume form $\Pi\left(d u_{i} \wedge d x_{i}\right)$. To find the volume forms $W_{1}$ and $W_{2}$ we need the next result.

Lemma 4.4. Consider the 2-form

$$
\omega=\sum \frac{d u_{i} \wedge d x_{i}}{c_{i}}
$$

in $T \mathbf{R}^{n}$. Then

$$
\frac{\omega^{n-1} \wedge(x d u) \wedge(x d x)}{W_{0}}=\frac{(n-1) !}{\Pi c_{i}} \sum c_{i} x_{i}^{2}
$$

and

$$
\frac{\omega^{n-2} \wedge(u d u) \wedge(u d x) \wedge(x d u) \wedge(x d x)}{W_{0}}=\frac{(n-2) !}{\Pi c_{i}} \sum_{i<j} c_{i} c_{j}\left(u_{i} x_{j}-u_{j} x_{i}\right)^{2}
$$

Proof. We prove the first equality; the second one is established similarly. The desired volume form is found as follows: choose a 2-dimensional subspace $L_{i}=$ $\operatorname{Span}\left(x_{i}, u_{i}\right)$; consider the area form $(x d u) \wedge(x d x)$ on $L_{i}$ and the volume form $\omega^{n-1}$ on $T \mathbf{R}^{n} / L_{i}$; multiply the two and sum over $i=1, \ldots, n$. The area form is $x_{i}^{2} d u_{i} \wedge d x_{i}$, and the symplectic volume form equals

$$
\frac{(n-1) ! \Pi_{j \neq i} d u_{j} \wedge d x_{j}}{\Pi_{j \neq i} c_{j}}
$$

The result follows by summation.

The previous lemma implies that, up to a constant,

$$
f_{t}=\frac{u^{2}\left(\sum b_{i} x_{i}^{2}\right)-\sum_{i<j} b_{i} b_{j}\left(u_{i} x_{j}-u_{j} x_{i}\right)^{2}}{u^{2}\left(\sum a_{i} x_{i}^{2}\right)}
$$

The denominator is the invariant function $\gamma$. Therefore the numerator is an integral too; denote it by $g_{t}$. It is easy to see that

$$
g_{t}=u^{2}\left(\sum b_{i} x_{i}^{2}\right)-\left(\sum b_{i} u_{i}^{2}\right)\left(\sum b_{j} x_{j}^{2}\right)+\left(\sum b_{i} u_{i} x_{i}\right)^{2}
$$


Consider the bilinear form in $\mathbf{R}^{n}$ :

$$
Q_{t}(a, b)=\sum \frac{a_{i} b_{i}}{1+a_{i} t}
$$

Since $x^{2}=1$ and $u x=0$, one has:

$$
\sum b_{i} x_{i}^{2}=1-Q_{t}(x, x), \quad \sum b_{i} u_{i}^{2}=u^{2}-Q_{t}(u, u), \quad \sum b_{i} u_{i} x_{i}=-Q_{t}(u, x) .
$$

It follows that

$$
g_{t}=Q_{t}(u, u)-Q_{t}(x, x) Q_{t}(u, u)+Q_{t}^{2}(u, x) .
$$

Following J. Moser $[8,, 9,10]$ one extracts $n$ integrals from this one-parameter family of invariant functions by expressing $g_{t}$ in partial fractions and taking the coefficient of $\left(1+a_{i} t\right)^{-1}$, divided by $a_{i}$, to be the $i$-th integral:

$$
F_{i}=\frac{u_{i}^{2}}{a_{i}}+\sum_{j \neq i} \frac{\left(u_{i} x_{j}-u_{j} x_{i}\right)^{2}}{a_{i}-a_{j}}
$$

Being expressed in terms of the coordinates $(y, v)$ on the original ellipsoid these functions coincide with the classical integrals of the geodesic flow as given in the quoted papers by Moser.

Remark. The geometrical meaning of the equation $g_{t}(x, u)=0$ is that the straight line in $\mathbf{R}^{n}$ through the point $x$ in the direction of $u$ is tangent to the quadric $Q_{t}=0$; this quadric is the image under the linear map $A$ of a quadric, confocal with the original ellipsoid $E-[8,9,10]$.

\section{Ellipsoids in the spherical and hyperbolic spaces}

It is natural to ask whether the methods of the previous sections are applicable to other Finsler metrics. A natural candidate would be a metric in a domain $D \subset \mathbf{R}^{n}$ with the following property: for every hypersurface $M \subset D$ there exists a transverse line field $\xi$ such that the Finsler geodesics on $M$ are the $\xi$-geodesics. The next result shows that if one restricts attention to the Riemannian case then the supply of such metrics is rather limited. Assume that the dimension $n$ is not less than 3 .

Theorem 5.1. A Riemannian metric $g$ enjoys the above property if and only if it is a metric of constant curvature whose geodesics are straight lines. In this case the transverse line field $\xi$ consists of the g-normals to a hypersurface.

Proof. Let a Finsler metric with the above property be given. First, we claim that the geodesics of this metric in $D$ are straight lines. Let $M$ be an affine hyperplane. 
Since the osculating 2-planes of every curve in $M$ lie in $M$ and cannot contain a transverse direction $\xi$, the Finsler geodesics in $M$ are straight lines.

Let $L$ be the Lagrangian, quadratic in the velocities $u$, defining the metric, and let $Q=L^{1 / 2}$. The non-parameterized extremals of $Q$ coincide with those of $L$.

We make use of Hamel's theorem that gives a necessary and sufficient condition for the extremals of a Lagrangian in a domain in $\mathbf{R}^{n}$, homogeneous of degree 1 , to be straight lines. This condition is: the matrix $Q_{x_{i} u_{j}}$ is symmetric - see [1]. In particular, if $M$ has the equation $x_{k}=$ const then the Hamel condition holds for all $i, j \neq k$. Since $n \geq 3$ the Hamel condition holds for all $i, j$, and the extremals of $Q$ in $\mathbf{R}^{n}$ are straight lines.

A Beltrami theorem states that a Riemannian metric whose geodesics are straight lines is a metric of constant curvature (see, e.g., [6]). To finish the proof one may use explicit formulas for the spherical or hyperbolic metric. We prefer, however, to derive a necessary and sufficient condition in terms of the Lagrangian.

The extremals $x(t)$ of the Lagrangian $Q$ on a hypersurface $M$ satisfy the EulerLagrange equation:

$$
Q_{u u} x^{\prime \prime}+Q_{u x} x^{\prime}-Q_{x}=C n(x)
$$

where $n(x)$ is a normal vector field to $M$ and $x^{\prime}=u$. Since $Q$ is homogeneous of degree 1, Euler's equation implies: $u Q_{u}=Q, u Q_{u u}=0$. Using Hamel's condition one obtains: $Q_{u x} u=Q_{x u} u=Q_{x}$; thus $Q_{u x} x^{\prime}-Q_{x}=0$. The condition imposed on the Finsler metric means that $x^{\prime \prime}$ is a linear combination of $\xi$ and $u$. Since $u Q_{u u}=0$ the Euler-Lagrange equation simplifies as follows:

$$
Q_{u u}(x, u) \xi(x)=C n(x) .
$$

Next we express this condition in terms of the Lagrangian $L$. One has:

$$
Q_{u u}=(1 / 2) L^{-3 / 2}\left(2 L L_{u u}-L_{u}^{T} L_{u}\right) ;
$$

thus the condition on the transverse field $\xi$ is:

$$
L_{u u}(x, u) \xi(x)-\frac{L_{u}(x, u) \cdot \xi(x)}{2 L(x, u)} L_{u}(x, u)=C n(x)
$$

for every $u$, orthogonal to $n(x)$.

Fix a point $x \in M$. If the metric is Riemannian then $L_{u u}$ is a symmetric matrix, say, $A$. Then $L_{u}=A u$ and $2 L=A u \cdot u$. Our condition reads:

$$
A \xi-\frac{A u \cdot \xi}{A u \cdot u} A u=C n
$$

for all $u$, orthogonal to $n$. This equality is satisfied by $\xi=A^{-1} n$ (and $C=1$ ), that is, by the $g$-normal to $M$ at $x$. The theorem is proved. 
We do not know whether the above theorem extends to all Finsler metrics. We conjecture this to be the case.

Use the standard models for the spherical and hyperbolic spaces: for the former it is, naturally, the sphere in the Euclidean space with the induced metric; for the latter it is one sheet of the 2-sheeted hyperboloid with the metric induced by the Lorentz metric of signature $(n, 1)$ in the ambient space. An ellipsoid in the spherical or hyperbolic space is the intersection of the sphere or hyperboloid with a positive-definite quadratic cone.

Using Theorem 5.1 and Property (iv) of exact transverse line fields from Section 3 , one can prove that the geodesic flow on the ellipsoid in the spherical or hyperbolic space is integrable (see [20] for a different approach). This proof essentially repeats the one given for the Euclidean case, and we do not dwell on it.

\section{Appendix: proof of Hamel's theorem}

For the reader's convenience we provide a proof of Hamel's theorem. This theorem is related to Hilbert's 4-th problem, integral and symplectic geometry - see [1].

Let $Q(x, u)$ be a Lagrangian, homogeneous of degree 1 , whose extremals are straight lines. The Euler-Lagrange equation reads:

$$
Q_{u u} x^{\prime \prime}+Q_{u x} x^{\prime}-Q_{x}=0
$$

where $x^{\prime}=u$. If the extremals are straight lines then $x^{\prime \prime}$ is proportional to $u$. Since $Q_{u}$ is homogeneous of degree 0 one has, by Euler's equation: $Q_{u u} u=0$. The Euler-Lagrange equation implies that $\sum_{k} Q_{u_{i} x_{k}} u_{k}=Q_{x_{i}}$ for all $i$. Differentiate with respect to $u_{j}$ to obtain:

$$
\sum_{k} Q_{u_{i} u_{j} x_{k}} u_{k}=Q_{x_{i} u_{j}}-Q_{x_{j} u_{i}}
$$

for all $i, j$. The left hand side is symmetric in $i, j$ while the right hand side is skew-symmetric. Therefore both vanish, and $Q_{x_{i} u_{j}}=Q_{x_{j} u_{i}}$.

Conversely, let the matrix $Q_{x_{i} u_{j}}$ be symmetric. Then $Q_{u x} u=Q_{x u} u=Q_{x}$, and $Q_{u x} u-Q_{x}=0$. The Euler-Lagrange equation implies that $Q_{u u} x^{\prime \prime}=0$. The matrix $Q_{u u}(x, u)$ is degenerate and its kernel is generated by the vector $u$. Thus $x^{\prime \prime}$ is proportional to $x^{\prime}$, and the extremals are straight lines.

Added in proof: After the submission of my paper I learned about the paper by V. Matveev and P. Topalov "Geodesic equivalence and integrability" (MPIM preprint, 1998) that contains a similar approach to the geodesic flow on the ellipsoid.

\section{Acknowledgments}

I am grateful to P. Tantalo for numerous stimulating discussions. Part of this work 
was done during the author's stay at MSRI and the University of Rennes; I am grateful to both institutions for their hospitality. The work was supported in part by an NSF grant.

\section{References}

[1] J. Alvarez, I. Gelfand, M. Smirnov, Crofton densities, symplectic geometry and Hilbert's Fourth Problem, 77-92. The Arnold-Gelfand Math. Sem., Birkhäuser, 1997.

[2] V. Arnold, A. Givental, Symplectic Geometry, 1-136. Encycl. of Math. Sci., Dynamical Systems, 4, Springer-Verlag, 1990.

[3] V. Arnold, Mathematical Methods of Classical Mechanics. Springer-Verlag, 1989.

[4] M. Audin, Courbes algébriques et systèmes intégrables: géodésiques des quadriques. Expo. Math. 12 (1994), 193-226.

[5] R. Brouzet, P. Molino, F. Turiel, Géométrie des systèmes bihamiltoniens. Indag. Math. 4(3) (1993), 269-296.

[6] H. Guggenheimer, Differential Geometry. McGraw-Hill, New York 1963.

[7] H. Knorrer, Geodesics on the ellipsoid, Invent. Math. 39 (1980), 119-143.

[8] J. Moser, Geometry of quadrics and spectral theory, Chern Symp. 1979, Springer-Verlag, 1980, pp. 147-188.

[9] J. Moser, Various aspects of integrable Hamiltonian systems. Progr. in Math. 8 Birhäuser, 1980.

[10] J. Moser, Integrable Hamiltonian systems and spectral theory. Lezioni Fermiane, Pisa, 1981.

[11] J. Moser, A. Veselov, Discrete versions of some classical integrable systems and factorization of matrix polynomials. Comm. Math. Phys. 139 (1991), 217-243.

[12] F. Magri, A simple model of the integrable Hamiltonian equations, J. Math. Phys. 19 (1978), 1156-1162.

[13] H. Rund, The Differential Geometry of Finsler Spaces. Springer-Verlag, 1959.

[14] R. Spatzier, Riemannian manifolds with completely integrable geodesic flows, Proc. Symp. Pure Math. 54 (1993), 599-608.

[15] S. Tabachnikov, Exact transverse line fields and projective billiards in a ball. GAFA 7 (1997), 594-608.

[16] S. Tabachnikov, Geometry of exact transverse line fields and projective billiards. To appear in Differential and symplectic topology of knots and curves, AMS "Adv. in Math. Sci." ser., Providence, 1999.

[17] S. Tabachnikov, Introducing projective billiards, Erg. Th. and Dyn. Syst. 17 (1997), 957-976.

[18] S. Tabachnikov, Billiards. SMF "Panoramas et Syntheses", N 1, 1995.

[19] S. Tabachnikov, Parameterized curves, Minkowski caustics, Minkowski vertices and conservative line fields, L'Enseign. Math. 43 (1997), 3-26.

[20] A. Veselov, Confocal surfaces and integrable billiards on the sphere and in the Lobachevsky space, J. Geom. Phys. 7 (1990), 81-107.

Serge Tabachnikov

University of Arkansas

Department of Mathematics

Fayetteville, AR 72701 USA

(Received: March 10, 1998) 